\title{
Postactivation Potentiation Biases Maximal Isometric Strength Assessment
}

\section{Leonardo Coelho Rabello Lima, ${ }^{1}$ Felipe Bruno Dias Oliveira, ${ }^{1}$ Thiago Pires Oliveira, ${ }^{1}$ Claudio de Oliveira Assumpção, ${ }^{1}$ Camila Coelho Greco, ${ }^{1}$ Adalgiso Croscato Cardozo, ${ }^{2}$ and Benedito Sérgio Denadai ${ }^{1}$}

\author{
${ }^{1}$ Human Performance Laboratory, Physical Education Department, São Paulo State University, Rio Claro, SP, Brazil \\ ${ }^{2}$ Biomechanics Laboratory, Physical Education Department, São Paulo State University, Rio Claro, SP, Brazil
}

Correspondence should be addressed to Leonardo Coelho Rabello Lima; leonardocrlima@gmail.com

Received 27 February 2014; Revised 20 June 2014; Accepted 1 July 2014; Published 15 July 2014

Academic Editor: Emanuele Marzetti

Copyright (C) 2014 Leonardo Coelho Rabello Lima et al. This is an open access article distributed under the Creative Commons Attribution License, which permits unrestricted use, distribution, and reproduction in any medium, provided the original work is properly cited.

\begin{abstract}
Postactivation potentiation (PAP) is known to enhance force production. Maximal isometric strength assessment protocols usually consist of two or more maximal voluntary isometric contractions (MVCs). The objective of this study was to determine if PAP would influence isometric strength assessment. Healthy male volunteers $(n=23)$ performed two five-second MVCs separated by a 180 -seconds interval. Changes in isometric peak torque (IPT), time to achieve it (tPTI), contractile impulse (CI), root mean square of the electromyographic signal during PTI (RMS), and rate of torque development (RTD), in different intervals, were measured. Significant increases in IPT $(240.6 \pm 55.7 \mathrm{~N} \cdot \mathrm{m}$ versus $248.9 \pm 55.1 \mathrm{~N} \cdot \mathrm{m})$, RTD $\left(746 \pm 152 \mathrm{~N} \cdot \mathrm{m} \cdot \mathrm{s}^{-1}\right.$ versus $\left.727 \pm 158 \mathrm{~N} \cdot \mathrm{m} \cdot \mathrm{s}^{-1}\right)$, and RMS $\left(59.1 \pm 12.2 \% \mathrm{RMS}_{\mathrm{MAX}}\right.$ versus $\left.54.8 \pm 9.4 \% \mathrm{RMS}_{\mathrm{MAX}}\right)$ were found on the second MVC. tPTI decreased significantly on the second MVC $(2373 \pm 1200 \mathrm{~ms}$ versus $2784 \pm 1226 \mathrm{~ms})$. We conclude that a first MVC leads to PAP that elicits significant enhancements in strength-related variables of a second MVC performed 180 seconds later. If disconsidered, this phenomenon might bias maximal isometric strength assessment, overestimating some of these variables.
\end{abstract}

\section{Introduction}

Postactivation potentiation (PAP) is a widely studied phenomenon that occurs when the neuromuscular system gets in a potentiated state after an applied stimulus [1]. The types of stimuli vary from isometric [2-4] and dynamic contractions $[5-7]$ to whole-body vibration $[8,9]$ and electrical stimulation [10] (i.e., posttetanic potentiation), which can lead to augmented power output in further activities like explosive contractions and sport-specific actions [1, 11, 12]. Some authors also found increases in maximal force production [13], even though most of the studies in the area present evidence for potentiated muscular power.

There are three major theories that explain the underlying physiological mechanisms of PAP [1]. One of the most consistent theories states that prior stimulation phosphorylates the myosin's regulatory light chains, moving them away from the myosin thick body and closer to the actin filaments [14], also increasing its sensitivity to $\mathrm{Ca}^{2+}$, which facilitate interactions within the sarcomeric apparatus [15]. Another consistent theory considers that preconditioning activities are responsible for increasing transmittance of excitation potentials in the synaptic junction and spinal cord levels [1618]. In order to try to better understand this phenomenon, a considerable number of studies assess electromyographic data in potentiated muscles $[2,16,18,19]$. A third theory suggests that a reduction in the pennation angle, induced by a potentiating stimulus, may contribute to increases in power and strength, once this change permits a more direct transmission from the muscle-fiber forces to the tendon. However, to our knowledge, there is only one study presenting solid evidence of this phenomenon [20].

In order to obtain optimal PAP response, some stimulation variables should be considered. There is an optimal 
interval between stimulation and assessment, which varies depending on the intensity and type of the stimulus [2123]. This interval must be long enough to promote recovery from acute metabolic fatigue and short enough to assess the muscle in a potentiated state (that disappears approximately 15 minutes after stimulation) $[1,24]$. In most studies, the interval between stimulation and assessment ranges between 3 and 8 minutes [2-6]. It is also known that neuromuscular factors such as strength levels, fiber type distribution, training level, and power/strength ratio play important roles on an individual's responsiveness to PAP $[1,25]$.

Strength production is one of the most important criterion measures adopted in studies that investigate the neuromuscular system [26-28]. A considerable number of studies use maximal isometric voluntary contraction (MVC) to assess muscular strength due to the number of variables that can be obtained from it (i.e., isometric peak torque (IPT), contractile impulse (CI), rate of torque development (RTD), etc.) [2, 29, 30]. For instance, RTD can be assessed in different time epochs from contraction onset (i.e., 10 to $250 \mathrm{~ms}$ ), providing information regarding the nature of the adaptation in explosive force. Indeed, the RTD assessed at early phase $(<100 \mathrm{~ms})$ is influenced by intrinsic muscle contractile properties and neural drive, whereas the late phase ( $>100 \mathrm{~ms}$ ) is influenced by muscle cross-sectional area, neural drive, and stiffness of tendon-aponeurosis complex [29]. Many strength assessment protocols adopt a predetermined number of MVCs separated by fixed rest intervals, using the contraction with the greatest isometric peak torque for analysis [29-32]. To the best of our knowledge, there are no studies reporting on which of the contractions is the most frequently analyzed. In that way, many of the isometric strength assessment protocols resemble potentiating protocols, using 3-5-second contractions separated by 30-60 seconds [29$34]$.

Considering the similarity between PAP stimulation and strength assessment protocols, our goal was to investigate whether PAP would bias isometric strength assessment (i.e., MVC), using a protocol involving two MVCs separated by a 180 -second rest interval. We hypothesized that a first MVC would potentiate the neuromuscular system, altering strength-related markers of a second MVC and, most importantly, influencing the selection of the analyzed contraction.

\section{Materials and Methods}

2.1. Subjects. Twenty-three males $(23.5 \pm 4 \mathrm{yr}, 177 \pm 8 \mathrm{~cm}$, $77.2 \pm 13.4 \mathrm{~kg}$, and $24.8 \pm 4.1 \mathrm{~kg} / \mathrm{m}^{2}$ ) volunteered for the study. One of the criteria for participation in the study was not to be engaged in any regular physical activity for the last 6 months, as well as not having any recent injury in the knee joint. The volunteers were asked not to take part in any physical activities other than those required on their daily routines during the study. All volunteers were informed about their rights as research subjects as well as the inherent risks of all the procedures by reading and signing an informed term of consent approved by the University Institutional Review Board for Human Subjects.
2.2. Experimental Design. The volunteers visited the laboratory on three occasions, each separated by 3-5 days. Two of these visits were used as familiarization sessions to lessen any effect of learning during subsequent strength testing. During these sessions, volunteers performed a nonspecific (8 min running at $8 \mathrm{~km} / \mathrm{h}$ ) and a specific warm-up (3 submaximal isometric contractions) before performing one MVC for the knee extensors in the dynamometer (Biodex System 3, Biodex Medical Systems, Shirley, NY). The third visit consisted of the experimental session, in which the volunteers followed the same warm-up procedure and, then, performed two MVCs for knee extensors in the isokinetic dynamometer to determine all criterion measures (IPT, RTD, RMS, and CI). T Seat set-up was recorded in the first familiarization session and reproduced in the following sessions. Volunteers were instructed to arrive at the laboratory in a rested and fully hydrated state, at least $3 \mathrm{~h}$ postprandially. They were also asked not to do any strenuous activity during the day prior to each test. All tests were performed in a climate-controlled $\left(21-22^{\circ} \mathrm{C}\right)$ laboratory at the same time of the day $( \pm 2 \mathrm{~h})$ to minimize the effects of diurnal biological variation on the results [35].

2.3. Familiarization Sessions. During the two familiarization trials, all volunteers were fully instructed and habituated to the test procedures in the dynamometer in order to avoid strength underestimation [36]. Prior to testing, they performed a standardized warm-up period of 8 min running at $8 \mathrm{~km} / \mathrm{h}$ followed by 3 submaximal isometric contractions of the knee extensors. Subsequently, each subject was carefully instructed to contract "as fast and forcefully as possible" when performing one five-second MVC.

2.4. Determination of the MVCs. The dynamometer and its specific computer software were used to measure MVC. Volunteers were placed in a sitting position and securely strapped into the test chair. Extraneous movement of the upper body was limited by two crossover shoulder harnesses and an abdominal belt. The trunk/thigh angle was $85^{\circ}$. The axis of the dynamometer was lined up with the right knee flexion-extension axis, and the lever arm was attached to the shank by a strap. The volunteers were asked to relax their legs so that passive determination of the effects of gravity on the limb and lever arm could be carried out. MVCs were performed for the knee extensors ( $\mathrm{m}$. quadriceps femoris) at a static knee joint angle of $75^{\circ}\left(0^{\circ}=\right.$ full extension $)$ [29, 30]. Two maximal isometric attempts were performed with rest periods of $180 \mathrm{~s}$ in between, which would allow proper recovery of the intracellular phosphocreatine reserves. The volunteers were instructed to extend their knees "as fast and forcefully as possible" [29] and each MVC was sustained for $5 \mathrm{~s}$.

2.5. Surface Electromyographic Recordings. The electromyographic (EMG) signal from the vastus lateralis muscle of the assessed limb was collected during all MVCs performed in the experimental session via disposable adherent $\mathrm{Ag} / \mathrm{AgCl}$ bipolar electrodes $(20 \mathrm{~mm}$ between poles) placed over the skin surface of the muscle and one simple reference electrode 
placed over the ulnar styloid process, both were connected to a preamplifier (100 times gain), following the SENIAM recommendations for electrode placement and EMG assessment [37], which were connected to a signal acquisition module (EMG System, Brazil) (20 times gain). Prior to electrode application, the skin was shaved, abraded, and cleansed with alcohol. The gain promoted by both the data acquisition system and the preamplifier enhanced the original biologic value 2000 times. Specific software was used to assess the EMG data with a $1000 \mathrm{~Hz}$ frequency.

2.6. Data Processing. The torque data of the contractions was obtained at a $1000 \mathrm{~Hz}$ frequency by a biological signal acquisition module (EMG System) synchronized with the isokinetic dynamometer. The data was filtered (Butterworth filter, low pass, 4th order, with a $15 \mathrm{~Hz}$ cut-off frequency) and analyzed in the MatLab 6.5 software (Mathworks, USA). The isometric peak torque (IPT) was considered the highest value in the torque-time curve, and the time point when it happened was also considered for analysis (tIPT). Contractile impulse (CI) was also calculated as the area under the torquetime curve, using trapezoidal integration $\left(\int_{a}^{b} f(x) d x\right)$. [29]. Rate of torque development (RTD) was obtained by the slope of the isometric contraction torque-time curve (i.e., $\Delta$ torque. $\Delta$ time $^{-1}$ ) across the time intervals of $0-30,0-50$, $0-100,0-150,0-200$, and $0-250 \mathrm{~ms}$ relative to the onset of contraction. Maximal rate of torque development $\left(\mathrm{RTD}_{\mathrm{MAX}}\right)$ was also calculated as the steepest point of the torque-time curve. The onset of muscle contraction was defined as the time point where the knee extensors torque exceeded the baseline by $2.5 \%$ of the baseline-to-peak difference [31].

The EMG data analyses were performed in the MatLab 6.5 software in which a high pass (Butterworth, high pass, 2nd order, with a $20 \mathrm{~Hz}$ cut-off frequency) and a low pass (Butterworth, low pass, 4th order, with a $500 \mathrm{~Hz}$ cut-off frequency) filters were applied. The average root mean square (RMS) value of the EMG signal obtained at a 0.5 -second interval during the MVC (i.e., 0.25 seconds before and 0.25 seconds after the IPT) was normalized by the peak RMS value obtained at the same time interval during EMG signal of the first experimental MVC. In that manner, all EMG values were expressed as percentages of the maximal RMS value $\left(\% \mathrm{RMS}_{\mathrm{MAX}}\right)$ of the first experimental contraction.

2.7. Statistical Analyses. All data are expressed as means \pm standard deviation (SD). Data normality was tested with the Shapiro-Wilk test. A one-way repeated-measures ANOVA was used to compare IPT obtained during familiarization sessions and at the first trial of the experimental session in order to identify possible familiarization and learning effects. Differences in all criterion measures (IPT, tIPT, CI, RTD, and EMG) obtained during the experimental session were identified using Student's $t$-tests for paired samples. Relationships between changes (2nd MVC and 1st MVC) in key dependent variables were assessed using Pearson's product moment correlation coefficients. The significance level was set at $P \leq 0.05$.

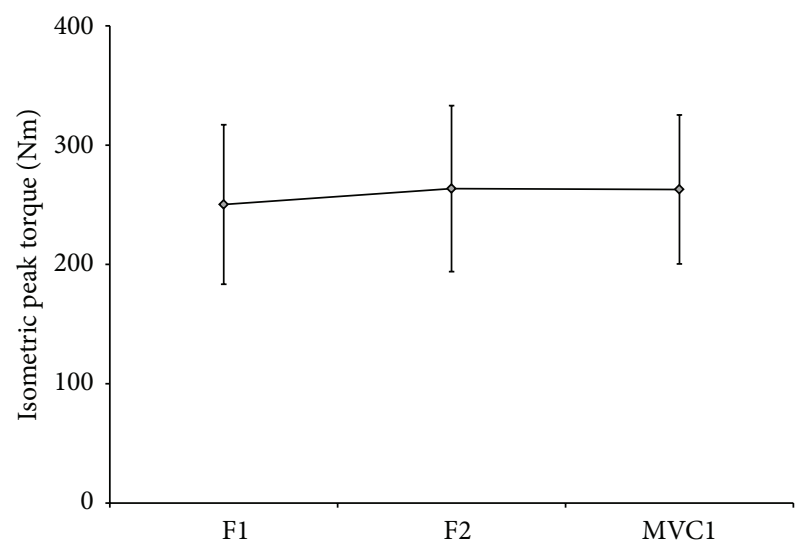

FIGURE 1: Mean (SD) isometric peak torque values obtained at the two familiarization sessions and the first maximal voluntary contraction. F1: first familiarization session; F2: second familiarization session; $\mathrm{MVCl}$ : first experimental maximal voluntary isometric contraction.

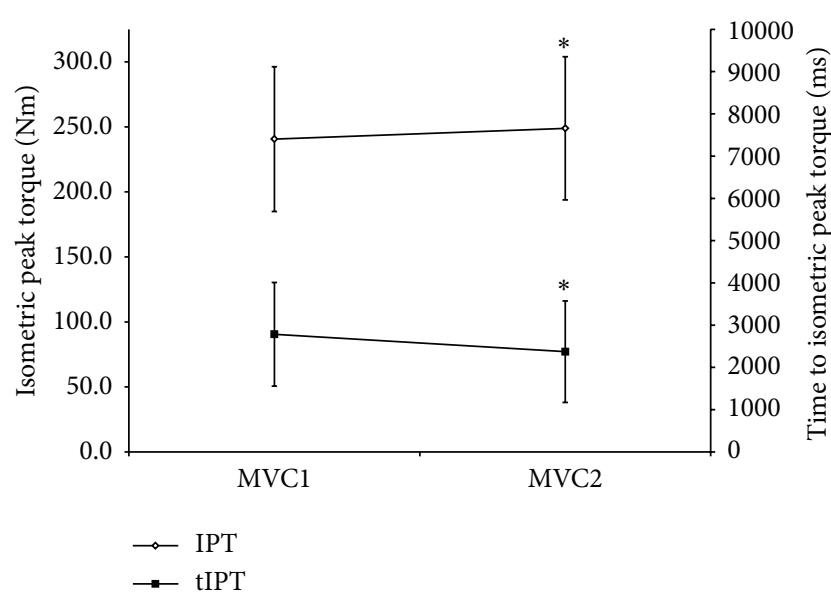

FIGURE 2: Mean (SD) isometric peak torque and time to reach peak torque values of the first and second maximal voluntary isometric contractions. MVC1: first maximal voluntary isometric contraction; MVC2: second maximal voluntary isometric contraction; * significantly different from the first contraction.

\section{Results}

The IPT values for both familiarization sessions were not significantly different than the IPT obtained at the first experimental session (Figure 1), which means that learning and adaptation to the tests did not bias the strength assessment.

IPT and tIPT values are expressed in Figure 2. IPT values of the second trial were significantly $(P=0.0003)$ higher than those obtained at the first trial $(240.6 \pm 55.7 \mathrm{~N} \cdot \mathrm{m}$ versus $248.9 \pm 55.1 \mathrm{~N} \cdot \mathrm{m})$. Also, the tPTI of the second trial $(2373 \pm$ $1200 \mathrm{~ms})$ was significantly $(P=0.02)$ lower than the tPTI of the first trial $(2784 \pm 1226 \mathrm{~ms})$. No significant alterations $(P>0.05)$ were found for CI (1st trial: $1086 \pm 274 \mathrm{~N} \cdot \mathrm{m}$ versus 2nd trial: $1106 \pm 286 \mathrm{~N} \cdot \mathrm{m})$.

There was a significant $(P=0.04)$ increase in the normalized RMS of the EMG signal of the vastus lateralis 


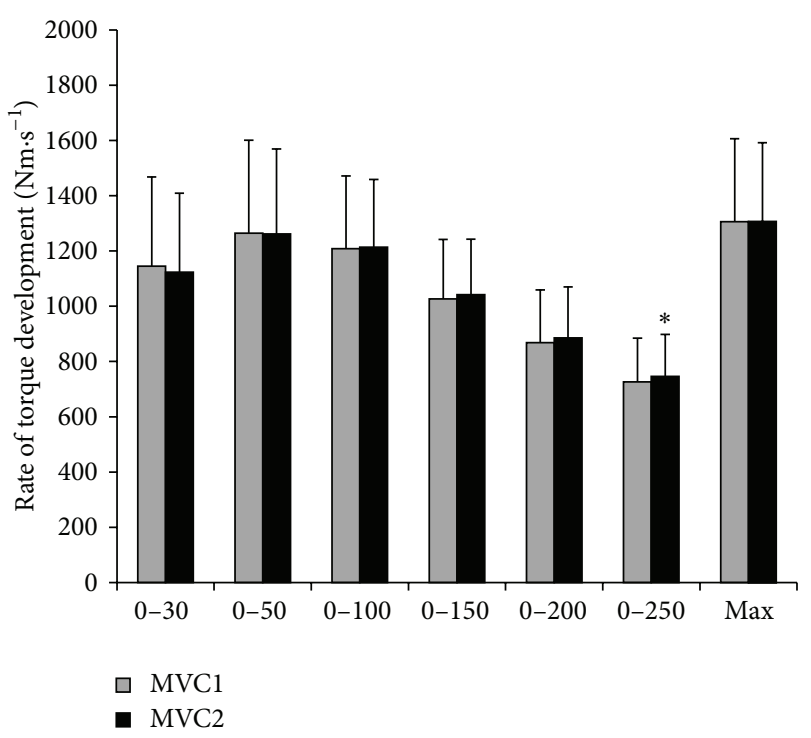

Figure 3: Mean (SD) rate of torque development in different intervals of the first and second maximal voluntary isometric contractions. MVC1: first maximal voluntary isometric contraction; MVC2: second maximal voluntary isometric contraction; * significantly different from the first contraction.

muscle during the second trial $\left(59.1 \pm 12.2 \% \mathrm{RMS}_{\mathrm{MAX}}\right)$ when compared to the first trial $\left(54.8 \pm 9.4 \% \mathrm{RMS}_{\mathrm{MAX}}\right)$. $\mathrm{RTD}_{\text {MAX }}$ did not present significant increases (1st trial: $1306 \pm 308 \mathrm{~N} \cdot \mathrm{m} \cdot \mathrm{s}^{-1}$ versus $2 \mathrm{nd}$ trial: $\left.1307 \pm 292 \mathrm{~N} \cdot \mathrm{m} \cdot \mathrm{s}^{-1}\right)$. However, $\operatorname{RTD}_{250}$ was significantly $(P=0.04)$ higher in the second trial $\left(746 \pm 152 \mathrm{~N} \cdot \mathrm{m} \cdot \mathrm{s}^{-1}\right)$ when compared to the first trial $\left(727 \pm 158 \mathrm{~N} \cdot \mathrm{m} \cdot \mathrm{s}^{-1}\right)$. RTD analyzed in all other intervals was not significantly increased at the second MVC (Figure 3). Additionally, a significant correlation $(r=0.54 ; P=0.01)$ was found for increases in IPT and EMG signal.

\section{Discussion}

The aim of the present study was to investigate if PAP would bias isometric strength assessment performed by a protocol consisting of two MVCs separated by a 180 -second rest interval. No learning effect was identified during the study, since IPT values for both familiarization sessions were not significantly different than those obtained at the first experimental MVC. Significant differences in IPT, tIPT, RMS, and RTD (relative to the $0-250 \mathrm{~ms}$ interval) were found between the two experimental MVCs, showing that PAP elicited increases/decreases in strength-related criterion measures.

It has been well established that familiarization sessions are an important methodological concern amongst studies that investigate isometric [38] and dynamic [39-41] muscular strength. Indeed, studies that investigate PAP [8, 42, 43] adopt familiarization sessions before the protocol in order to avoid over- and underestimation in strength assessment [39]. Although most studies use single familiarization before engaging in experimental sessions and applying potentiating stimuli $[8,42]$, we chose to add an extra session to the familiarization protocol, considering the data from Wallerstein et al. [38], which showed stability in IPT values only after a third familiarization session in the elderly (i.e., the IPT of the fourth session was not different from the third). However, adopting IPT as criteria for stabilization, we found that either one or two familiarization sessions were enough to attend to this purpose in young adults. To our knowledge, there are no studies that investigated how many familiarization sessions are necessary to reach IPT stability amongst young males. Nevertheless, reproducibility and validity of strength assessment in isokinetic dynamometers have been otherwise described [44, 45].

PAP is known to significantly enhance muscular power assessed through isokinetic contractions [2], jumps [2, 3, 5], and other athletic activities [11]. However, in the present study, a previous MVC led to $3.5 \%$ increases in IPT of a second MVC performed 180 seconds later. Few are the studies that investigated the influence of PAP on isometric contractions [42, 46, 47]. Fukutani et al. [46] and Miyamoto et al. [47], for instance, found increased twitch isometric torque values after isokinetic contractions. Both of these studies showed that PAP induced by dynamic activities can enhance maximal force production. This enhancement in maximal isometric strength can be partially explained by changes in the pennation angle, one of the proposed mechanisms for PAP [20]. Moreover, a 4,5\% increase in the activation of the vastus lateralis was also identified during this study, which could have happened due to increased transmittance in the synaptic junctions of the motoneuron $[16,17]$ or decreased synaptic failures, leading to the recruitment of a greater number of motor units [18]. Indeed, a significant moderate correlation was found between the increases in IPT and RMS of the vastus lateralis, contributing to this rationale. However, the common variance $\left(r^{2}=0.29\right)$ was low, which could not provide a cause-effect relationship between the increases of IPT and RMS.

Although IPT increased significantly in the second MVC, CI values were not significantly different between each other, which means that PAP might elicit increases in maximal force production but not in its maintenance whatsoever. However, adding to the knowledge that PAP increases explosive muscular actions [2-7], the tPTI decreased significantly during the second MVC. This alteration might be explained by the phosphorylation of the myosin light chain, rendering structural changes within the sarcomeres and augmented sensitivity to $\mathrm{Ca}^{2+}$, as reported by Szczesna et al. [14].

Besides tPTI, the main variable adopted in this study to investigate explosive force was RTD (maximal and on fixed intervals). Although IPT increased after the first MVC, maximal RTD was not influenced by the PAP elicited by a prior contraction. Other authors identify a potentiating effect caused by dynamic contractions on the RTD [42, 43]. For instance, Gilbert and Lees [42] found that RTD measured by MVC is potentiated after both maximal strength and power exercises. They also showed that a longer period ( 15-20 minutes) than that adopted in the present study might be necessary for the potentiation of RTD after maximal strength 
exercise (1RM squat). Baudry and Duchateau [43] also found increased maximal RTD after a six-second ballistic MVC of the thumb adductor showing, however, that maximal RTD measured by twitch contractions might be greatly influenced by PAP, since it does not depend on metabolic factors such as complete restoration of the intracellular phosphocreatine reserves within the muscle. On the other hand, Bazett-Jones et al. [48] found that maximal RTD was not enhanced one minute after a potentiating protocol consisting of three dynamic contractions with a load of $90 \% 1 \mathrm{RM}$. Instead, RTD decreased significantly after the conditioning protocol.

To the best of our knowledge, there are no studies investigating the effects of PAP on the RTD measured in classically adopted fixed intervals $(0-30,0-50,0-100,0-200$, and $0-250 \mathrm{~ms}$ ) $[29,30]$. The results obtained in our study showed that, of all analyzed intervals, the only one to respond to PAP was $0-250 \mathrm{~ms}$. Although all RTD measurements represent explosive strength, its manifestation in early intervals (i.e., 0-30 to $0-100 \mathrm{~ms}$ ) seems to be related to neural factors such as motoneuron recruitment, firing frequencies, and $\mathrm{Ca}^{2+}$ kinetics, while latter intervals seem to be more related to maximal strength production [29]. In that way, the increases in RTD in the $0-250 \mathrm{~ms}$ interval are coherent with the increases in IPT and EMG observed in our study.

Validity and reliability are paramount in strength assessment $[49,50]$. One of the biggest concerns in investigations that involve sensible strength variables such as RTD is to ensure proper placement and fixation of the subjects on the strength measuring device [29], as well as the maintenance of the exact same testing conditions. With that assured, many researchers adopt the MVC with the greatest IPT value for analyses [29-32], disregarding potential PAP and/or fatigue effects during the protocol. Our data showed that, using a protocol consisting of two MVCs separated by a 180 -second rest interval, PAP might occur increasing IPT values and biasing the selection of the analyzed MVC. Moreover, in investigations that consider explosive strength and EMG signal, the potentiating effects of a first MVC might also influence important variables like RTD measured in different time intervals and RMS values. Notwithstanding, there are other factors that might affect PAP. It is known that fiber type distribution, strength levels, and power/strength ratio are important factors that contribute to an individuals' responsiveness to PAP $[1,25]$. Therefore, in study models that involve neuromuscular training, not only could PAP bias initial strength assessment, but also influence post-training measurements, eliciting higher increases in strength-related variables for groups that experienced training interventions. This increased PAP responsiveness could be responsible for magnifying the effects of training, as the control group would remain in the same training state, responding similarly to PAP.

\section{Conclusion}

We conclude that the strength assessment protocol we adopted in the present study might be biased by PAP, since a first MVC renders the neuromuscular system in a potentiated state, while the rest intervals allow enough time for the ATP$\mathrm{CP}$ system to partially recover, resulting in an enhanced strength response. Moreover, it is important to note that study protocols involving strength training might also be biased by PAP, since well-trained individuals respond better to this phenomenon. Therefore, we suggest that researchers and practitioners consider potential PAP effects on isometric strength assessment protocols and take extra care in the analyses process to avoid confounding data.

\section{Conflict of Interests}

The authors declare that there is no conflict of interests regarding the publication of this paper.

\section{Acknowledgments}

The authors would like to thank Coordenação de Aperfeiçoamento de Pessoal de Nível Superior (CAPES) and Conselho Nacional de Desenvolvimento Científico (CNPq) for the financial support.

\section{References}

[1] N. A. Tillin and D. Bishop, "Factors modulating post-activation potentiation and its effect on performance of subsequent explosive activities," Sports Medicine, vol. 39, no. 2, pp. 147-166, 2009.

[2] D. N. French, W. J. Kraemer, and C. B. Cooke, "Changes in dynamic exercise performance following a sequence of preconditioning isometric muscle actions," The Journal of Strength \& Conditioning Research, vol. 17, no. 4, pp. 678-685, 2003.

[3] J. M. Berning, K. J. Adams, M. Debeliso, P. G. Sevene-Adams, C. Harris, and B. A. Stamford, "Effect of functional isometric squats on vertical jump in trained and untrained men," Journal of Strength and Conditioning Research, vol. 24, no. 9, pp. 2285$2289,2010$.

[4] J. I. Esformes, M. Keenan, J. Moody, and T. M. Bampouras, "Effect of different types of conditioning contraction on upper body postactivation potentiation," Journal of Strength and Conditioning Research, vol. 25, no. 1, pp. 143-148, 2011.

[5] K. R. Weber, L. E. Brown, J. W. Coburn, and S. M. Zinder, "Acute effects of heavy-load squats on consecutive squat jump performance," The Journal of Strength \& Conditioning Research, vol. 22, no. 3, pp. 726-730, 2008.

[6] E. D. Hanson, S. Leigh, and R. G. Mynark, "Acute effects of heavy- and light-load squat exercise on the kinetic measures of vertical jumping," Journal of Strength and Conditioning Research, vol. 21, no. 4, pp. 1012-1017, 2007.

[7] C. J. Mitchell and D. G. Sale, "Enhancement of jump performance after a 5-RM squat is associated with postactivation potentiation," European Journal of Applied Physiology, vol. 111, no. 8, pp. 1957-1963, 2011.

[8] D. J. Cochrane, S. R. Stannard, E. C. Firth, and J. Rittweger, "Acute whole-body vibration elicits post-activation potentiation," European Journal of Applied Physiology, vol. 108, no. 2, pp. 311-319, 2010.

[9] M. Jordan, S. Norris, D. Smith, and W. Herzog, "Acute effects of whole-body vibration on peak isometric torque, muscle twitch torque and voluntary muscle activation of the knee extensors," 
Scandinavian Journal of Medicine and Science in Sports, vol. 20, no. 3, pp. 535-540, 2010.

[10] S. Baudry, M. Klass, and J. Duchateau, "Postactivation potentiation of short tetanic contractions is differently influenced by stimulation frequency in young and elderly adults," European Journal of Applied Physiology, vol. 103, no. 4, pp. 449-459, 2008.

[11] L. P. Kilduff, D. J. Cunningham, N. J. Owen, D. J. West, R. M. Bracken, and C. J. Cook, "Effect of postactivation potentiation on swimming starts in international sprint swimmers," The Journal of Strength \& Conditioning Research, vol. 25, no. 9, pp. 2418-2423, 2011.

[12] E. E. Linder, J. H. Prins, N. M. Murata, C. DeRenne, C. F. Morgan, and J. R. Solomon, "Effects of preload 4 repetition maximum on 100-m sprint times in collegiate women," Journal of Strength and Conditioning Research, vol. 24, no. 5, pp. 11841190, 2010.

[13] M. A. B. Batista, C. Ugrinowitsch, H. Roschel, R. Lotufo, M. D. Ricard, and V. A. A. Tricoli, "Intermittent exercise as a conditioning activity to induce postactivation potentiation," Journal of Strength and Conditioning Research, vol. 21, no. 3, pp. 837-840, 2007.

[14] D. Szczesna, J. Zhao, M. Jones, G. Zhi, J. Stull, and J. D. Potter, "Phosphorylation of the regulatory light chains of myosin affects $\mathrm{Ca}^{2+}$ sensitivity of skeletal muscle contraction," Journal of Applied Physiology, vol. 92, no. 4, pp. 1661-1667, 2002.

[15] D. Szczesna, "Regulatory light chains of striated muscle myosin. Structure, function and malfunction," Current Drug TargetsCardiovascular and Haematological Disorders, vol. 3, no. 2, pp. 187-197, 2003.

[16] J.-. Gossard, M. K. Floeter, Y. Kawai, R. E. Burke, T. Chang, and S. J. Schiff, "Fluctuations of excitability in the monosynaptic reflex pathway to lumbar motoneurons in the cat," Journal of Neurophysiology, vol. 72, no. 3, pp. 1227-1239, 1994.

[17] H. R. Lüscher, P. Ruenzel, and E. Henneman, "Composite EPSPs in motoneurons of different sizes before and during PTP: implications for transmission failure and its relief in Ia projections," Journal of Neurophysiology, vol. 49, no. 1, pp. 269289, 1983.

[18] G. D. S. Hirst, S. J. Redman, and K. Wong, "Post-tetanic potentiation and facilitation of synaptic potentials evoked in cat spinal motoneurons," Journal of Physiology, vol. 321, pp. 97-109, 1981.

[19] N. Shima, C. J. McNeil, and C. L. Rice, "Mechanomyographic and electromyographic responses to stimulated and voluntary contractions in the dorsiflexors of young and old men," Muscle and Nerve, vol. 35, no. 3, pp. 371-378, 2007.

[20] K. Mahlfeld, J. Franke, and F. Awiszus, "Postcontraction changes of muscle architecture in human quadriceps muscle," Muscle and Nerve, vol. 29, no. 4, pp. 597-600, 2004.

[21] M. R. McCann and S. P. Flanagan, "The effects of exercise selection and rest interval on postactivation potentiation of vertical jump performance," Journal of Strength and Conditioning Research, vol. 24, no. 5, pp. 1285-1291, 2010.

[22] L. P. Kilduff, N. Owen, H. Bevan, M. Bennett, M. I. C. Kingsley, and D. Cunningham, "Influence of recovery time on postactivation potentiation in professional rugby players," Journal of Sports Sciences, vol. 26, no. 8, pp. 795-802, 2008.

[23] A. Chaouachi, N. Poulos, F. Abed et al., "Volume, intensity, and timing of muscle power potentiation are variable," Applied Physiology, Nutrition and Metabolism, vol. 36, no. 5, pp. 736-747, 2011.

[24] R. P. Lowery, N. M. Duncan, J. P. Loenneke et al., “The effects of potentiating stimuli intensity under varying rest periods on vertical jump performance and power," Journal of Strength and Conditioning Research, vol. 26, no. 12, pp. 3320-3325, 2012.

[25] M. A. B. Batista, H. Roschel, R. Barroso, C. Ugrinowitsch, and V. Tricoli, "Influence of strength training background on postactivation potentiation response," Journal of Strength and Conditioning Research, vol. 25, no. 9, pp. 2496-2502, 2011.

[26] K. M. Winters-Stone, J. Dobek, J. A. Bennett, L. M. Nail, M. C. Leo, and A. Schwartz, "The effect of resistance training on muscle strength and physical function in older, postmenopausal breast cancer survivors: a randomized controlled trial," Journal of Cancer Survivorship, vol. 6, no. 2, pp. 189-199, 2012.

[27] K. R. Thompson, A. E. Mikesky, R. E. Bahamonde, and D. B. Burr, "Effects of physical training on proprioception in older women," Journal of Musculoskeletal Neuronal Interactions, vol. 3, no. 3, pp. 223-231, 2003.

[28] A. W. Frank, A. L. Lorbergs, P. D. Chilibeck, J. P. Farthing, and S. A. Kontulainen, "Muscle cross sectional area and grip torque contraction types are similarly related to PQCT derived bone strength indices in the radii of older healthy adults," Journal of Musculoskeletal Neuronal Interactions, vol. 10, no. 2, pp. 136-141, 2010.

[29] P. Aagaard, E. B. Simonsen, J. L. Andersen, P. Magnusson, and P. Dyhre-Poulsen, "Increased rate of force development and neural drive of human skeletal muscle following resistance training," Journal of Applied Physiology, vol. 93, no. 4, pp. 13181326, 2002.

[30] L. L. Andersen and P. Aagaard, "Influence of maximal muscle strength and intrinsic muscle contractile properties on contractile rate of force development," European Journal of Applied Physiology, vol. 96, no. 1, pp. 46-52, 2006.

[31] L. L. Andersen, J. L. Andersen, M. K. Zebis, and P. Aagaard, "Early and late rate of force development: differential adaptive responses to resistance training?" Scandinavian Journal of Medicine and Science in Sports, vol. 20, no. 1, pp. e162-e169, 2010.

[32] F. B. D. Oliveira, A. S. C. Oliveira, G. F. Rizatto, and B. S. Denadai, "Resistance training for explosive and maximal strength: effects on early and late rate of force development," Journal of Sports Science and Medicine, vol. 12, no. 3, pp. 402408, 2013.

[33] T. C. Chen, K. Nosaka, and J. H. Tu, "Changes in running economy following downhill running," Journal of Sports Sciences, vol. 25, no. 1, pp. 55-63, 2007.

[34] L. C. R. de Lima and B. S. Denadai, "The repeated bout effect: a comparison between upper and lower limbs," Motriz: Revista de Educação Física, vol. 17, no. 4, pp. 738-747, 2011.

[35] G. Atkinson and T. Reilly, "Circadian variation in sports performance," Sports Medicine, vol. 21, no. 4, pp. 292-312, 1996.

[36] A. S. Oliveira, R. B. Corvino, M. Gonçalves, F. Caputo, and B. S. Denadai, "Effects of a single habituation session on neuromuscular isokinetic profile at different movement velocities," European Journal of Applied Physiology, vol. 110, no. 6, pp. 11271133, 2010.

[37] H. J. Hermens, B. Freriks, C. Disselhorst-Klug, and G. Rau, "Development of recommendations for SEMG sensors and sensor placement procedures," Journal of Electromyography \& Kinesiology, vol. 10, no. 5, pp. 361-374, 2000.

[38] L. F. Wallerstein, R. Barroso, V. Tricoli, M. T. Mello, and C. Ugrinowitsch, "The influence of familiarization sessions on the stability of ramp and ballistic isometric torque in older adults," Journal of Aging and Physical Activity, vol. 18, no. 4, pp. 390-400, 2010. 
[39] J. W. Coburn, T. J. Housh, M. H. Malek et al., "Neuromuscular responses to three days of velocity-specific isokinetic training," Journal of Strength and Conditioning Research, vol. 20, no. 4, pp. 892-898, 2006.

[40] I. Urzica, V. Tiffreau, S. Popielarz, B. Duquesnoy, and A. Thevenon, "Isokinetic trunk strength testing in chronic low back pain. The role of habituation and training to improve measures," Annales de Readaptation et de Medecine Physique, vol. 50, no. 5, pp. 271-274, 2007.

[41] K. M. Calder and D. A. Gabriel, "Adaptations during familiarization to resistive exercise," Journal of Electromyography and Kinesiology, vol. 17, no. 3, pp. 328-335, 2007.

[42] G. Gilbert and A. Lees, "Changes in the force development characteristics of muscle following repeated maximum force and power exercise," Ergonomics, vol. 48, no. 11-14, pp. 15761584, 2005.

[43] S. Baudry and J. Duchateau, "Postactivation potentiation in a human muscle: effect on the rate of torque development of tetanic and voluntary isometric contractions," Journal of Applied Physiology, vol. 102, no. 4, pp. 1394-1401, 2007.

[44] N. A. Maffiuletti, M. Bizzini, K. Desbrosses, N. Babault, and U. Munzinger, "Reliability of knee extension and flexion measurements using the Con-Trex isokinetic dynamometer," Clinical Physiology and Functional Imaging, vol. 27, no. 6, pp. 346-353, 2007.

[45] J. Toonstra and C. G. Mattacola, "Test-retest reliability and validity of isometric knee flexion and extension measurement using three methods of assessing muscle strength," Journal of Sport Rehabilitation, Technical report 7, pp. 1-5, 2013.

[46] A. Fukutani, N. Myiamoto, H. Kanehisa, T. Yanai, and Y. Kawakami, "Potentiation of isokinetic torque is velocitydependent following na isometric conditioning contraction," Springerplus, vol. 2, p. 554, 2013.

[47] N. Miyamoto, H. Kanehisa, T. Fukunaga, and Y. Kawakami, "Effect of postactivation potentiation on the maximal voluntary isokinetic concentric torque in humans," Journal of Strength and Conditioning Research, vol. 25, no. 1, pp. 186-192, 2011.

[48] D. M. Bazett-Jones, J. B. Winchester, and J. M. McBride, "Effect of potentiation and stretching on maximal force, rate of force development, and range of motion," Journal of Strength and Conditioning Research, vol. 19, no. 2, pp. 421-426, 2005.

[49] K. Lienhard, S. P. Lauermann, D. Schneider, J. F. Item-Glatthorn, N. C. Casartelli, and N. A. Maffiuletti, "Validity and reliability of isometric, isokinetic and isoinertial modalities for the assessment of quadriceps muscle strength in patients with total knee arthroplasty," Journal of Electromyography and Kinesiology, vol. 23, no. 6, pp. 1283-1288, 2013.

[50] S. C. Webber and M. M. Porter, "Reliability of ankle isometric, isotonic, and isokinetic strength and power testing in older women," Physical Therapy, vol. 90, no. 8, pp. 1165-1175, 2010. 

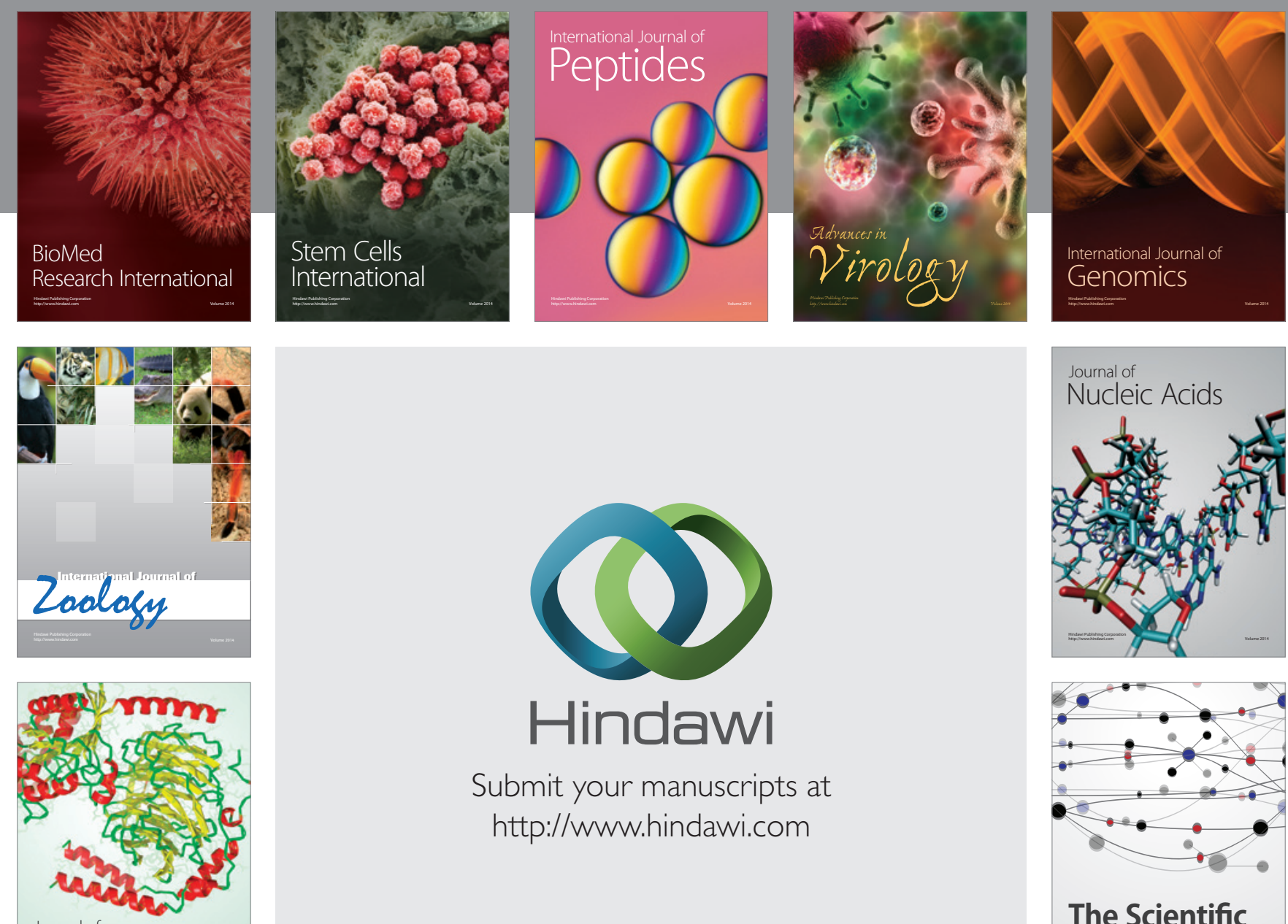

Submit your manuscripts at

http://www.hindawi.com

Journal of
Signal Transduction
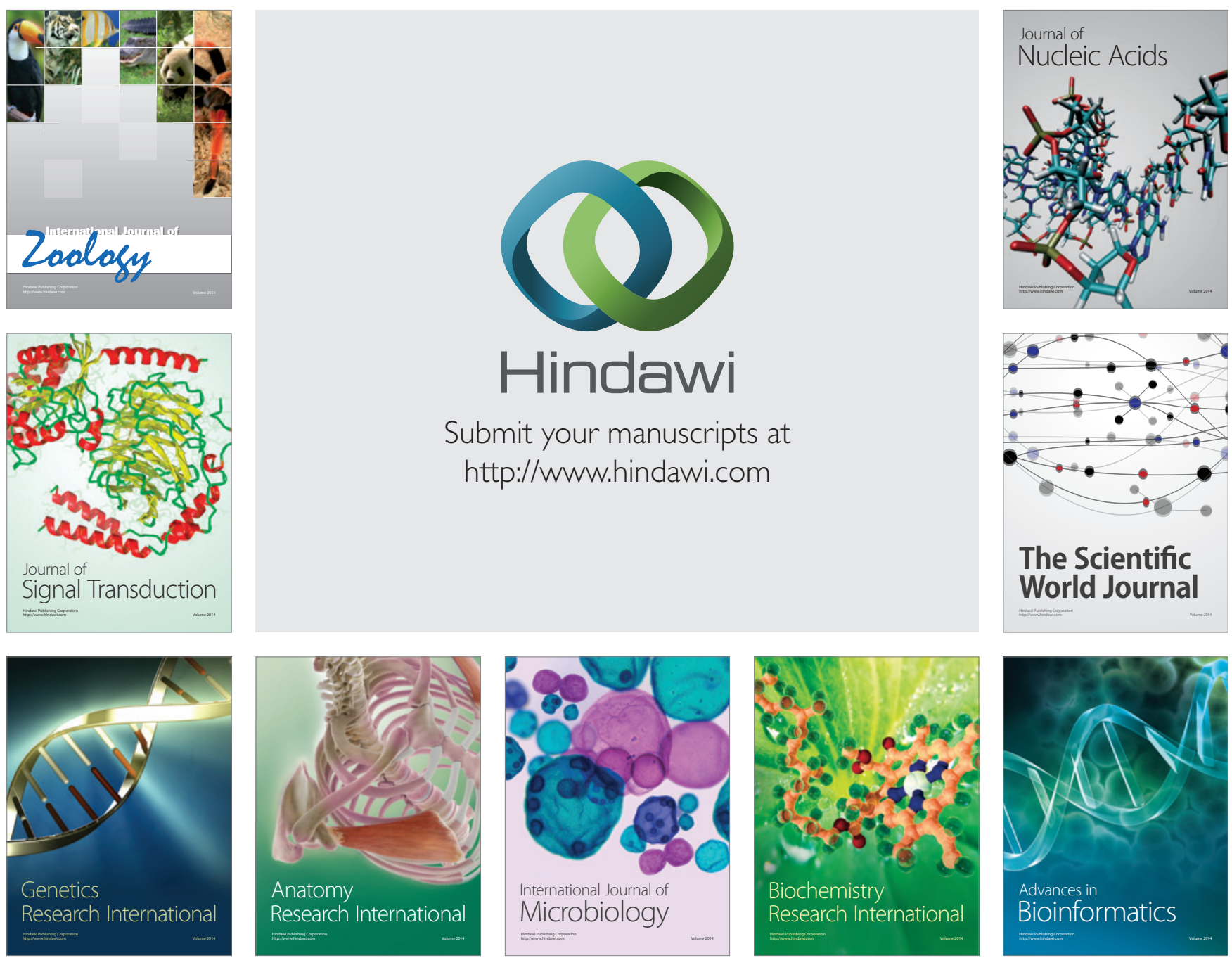

The Scientific World Journal
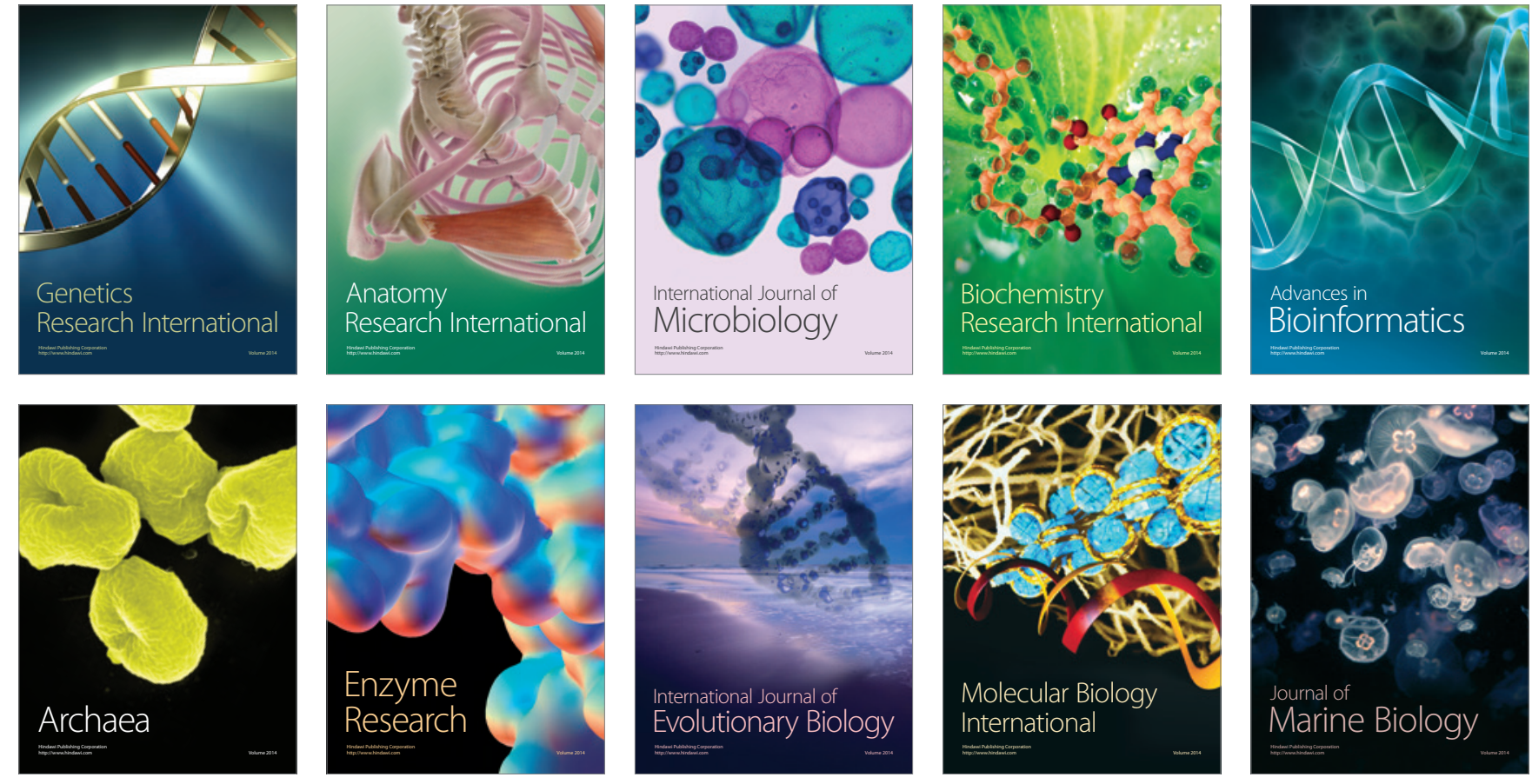